\title{
Die schwierige Finanzierung des Journalismus
}

\author{
Marie Luise Kiefer
}

Der Beitrag greift die aktuelle Frage einer Finanzierung des Journalismus auf, deren privatwirtschaftliche Lösung als mediale Querfinanzierung aus dem Werbemarkt zumindest als allgemeines Muster offenbar einem Ende zugeht. Die Suche nach einem dritten Weg zwischen den bestehenden Alternativen privatwirtschaftlich vs. öffentlich-rechtlich erfolgt in mebreren Schritten. Voraussetzung einer medienunabhängigen Journalismusfinanzierung ist die Unterscheidung von Journalismus und Medien, die mit institutionentheoretischen Ansätzen erfolgt und Journalismus als die demokratietheoretisch fundamentale Institution ausweist. Eine öffentliche Finanzierung dieser Institution, für die hier plädiert wird, setzt deren Formalisierung und staatsferne soziale Organisation voraus. Den Weg dorthin weist die institutionenökonomische Theorie der Commons als Selbstorganisation, das konkrete Modell dafür liefert das soziologische Professionskonzept als eine Form der Commons. Der Staat ist vor allem in der Rolle des Ermöglichers der skizzierten Selbstorganisationsprozesse des Journalismus gefordert, die notwendige Definitions- und Abgrenzungsarbeit ist von Journalismustheorie und -praxis zu leisten. Die mit dem Kollektivgutcharakter journalistischer Dienstleistung begründbare öffentliche Finanzierung wird als eine vom Staat zu organisierende Finanzierung aus verschiedenen Quellen entwickelt.

Schlagwörter: Journalismustheorie, Institutionenökonomik, Theorie der Commons, Profession, Journalismusorganisation, Journalismusfinanzierung

\section{Problemaufriss}

Eine mediale Querfinanzierung des Journalismus aus dem Werbemarkt wird offenbar immer schwieriger. Sinkende Werbeeinahmen der gedruckten Zeitungen vor allem durch Verlust der lukrativen Rubrikenwerbung an das Internet, Konkurrenz im Internet auch durch direkte Werbemöglichkeiten der Unternehmen für ihre Produkte und Dienstleistungen, geringe und für nicht spezialisierte Angebote offenbar eher sinkende (Breunig 2005) Zahlungsbereitschaft der Internetnutzer sowie Kostenwettbewerb aus Sicht der Leser zwischen Print- und Online-Ausgaben einer Zeitung (Mögerle 2009) sind Rahmenbedingungen, die den Ausblick in die Zukunft journalistischer Medien, hier am Beispiel der Tageszeitung als Prototyp exemplifiziert, eher düster erscheinen lassen ${ }^{1}$. Verschärft wird die Situation durch die aktuelle Wirtschaftskrise. Hinzu kommt, dass der Bereich der Medien, auch der journalistischen Medien, vom gesellschaftlichen Metatrend der Ökonomisierung als einer „,Überformung' konkurrierender Soziallogiken durch die wirtschaftliche“ (Richter 2009: 216) nicht verschont blieb. Das Interesse von Kapitalinvestoren an Medienunternehmen ist dafür lediglich ein Indikator. Mit Blick auf die Entwicklung in den USA, aber nicht nur dort, halten Weichert/Kramp (2009: 6) fest: „Qualitätsjournalismus ist in der Perspektive großer Konzerne nur noch ein Produkt unter

1 Für alle vorwiegend werbefinanzierten Medien, also auch Zeitschriften, privater Hörfunk und privatwirtschaftlich organisiertes Fernsehen, haben sich die wirtschaftlichen Rahmenbedingungen „derart verschlechtert, dass die Finanzierbarkeit privater Medienangebote überall in Frage gestellt ist“ (Siebenhaar 2010: 8). „Die klassische Medienindustrie sitzt in der Werbefalle“ (ebenda: 7) und sucht nach neuen Erlösmodellen. 
vielen, das künftig noch stärker den oftmals volatilen Marktbedingungen unterliegt ${ }^{\text {“2 }}$. Dass auch die deutschen Verlagshäuser ihrer veränderten Marktsituation zunehmend durch Absenkung der Redaktionskosten zu begegnen versuchen, macht u. a. die aktuelle Konzentrationsanalyse von Röper (2010) deutlich. ${ }^{3}$

Für die Zukunft des Journalismus und journalistischer Medien fügen sich diese und weitere Facetten jedenfalls zu einem Szenario, das Nachdenken über ihre Finanzierung gebietet, sofern man davon ausgeht, dass beide für demokratische Gesellschaften unverzichtbar sind. Damit wären hier zwei zentrale Fragen zu klären:

1. Sind Journalismus und journalistische Medien für demokratische Gesellschaften unverzichtbar und wenn ja, warum?

2. Lässt sich ein Modell öffentlicher Finanzierung von Journalismus und journalistischen Medien entwickeln, das mit dem Gebot der Staatsferne und der Pressefreiheit vereinbar ist?

Die Frage, ob zu einer öffentlichen Finanzierung alternative Modelle einer wirtschaftlichen Sicherung von Journalismus diskutiert bzw. erprobt werden und wenn ja, welche, muss hier aus Platzgründen unberücksichtigt bleiben. ${ }^{4}$ Generelle Lösungen scheinen bislang jedenfalls nicht in Sicht. So glauben Downie/Schudson in einem Ende 2009 vorgelegten Bericht „The Reconstruction of American Journalism“ nicht, dass Journalismus in Zukunft allein aus einem privatwirtschaftlichen Geschäftsmodell finanziert werden kann. Sie erarbeiten daher einen umfangreichen Katalog an privaten und öffentlichen Teilfinanzierungsmöglichkeiten, die sie allerdings nicht $\mathrm{zu}$ einem Finanzierungsgesamtkonzept verbinden. So bleibt auch ihr Appell an die amerikanische Gesellschaft, kollektiv Verantwortung für einen unabhängigen Journalismus zu übernehmen (vgl. ebenda: 75) eher vage.

Gerade das Papier von Downie/Schudson macht deutlich, dass ein Konzept verlässlicher Finanzierung des Journalismus, sofern man diesen für demokratienotwendig hält, als eine kollektiv organisierte und das heißt durch den Gesetzgeber geregelte Lösung gedacht werden muss. Diese ansatzweise zu entwickeln, wird hier unter Rückgriff auf institutionalistische und professionssoziologische Ansätze versucht:

Ausgangspunkt ist die institutionentheoretisch begründete Trennung von Journalismus und Medien, die Journalismus als die im Kontext einer demokratischen Ordnung fundamentale Institution ausweist (2.). Diese Trennung wirft die Frage nach einer sozialen Organisation von Journalismus auf, die medienunabhängig als Andockmöglichkeit dienen kann für kommunikationspolitisches Handeln, das eine kollektive Finanzierung ja impliziert. Dafür bietet sich ein Rückgriff auf das soziologische Konzept der Profession als autonome Kollegialorganisation an. Nicht nur wird Journalismus traditionell zu den Professionen zumindest im weiteren Sinne gezählt; die zentrale theoretische Verbindungsbrücke ist, dass Professionen generell als Institutionen im hier verwendeten Sinne gelten (3.).

Mit dem Professionskonzept als Organisationsvorlage stellt sich die weitere Frage, wie die Professionsbildung der „verspäteten Profession“ (Neverla 1998) unter Berück-

2 Nach Schätzungen des Berichts zur Lage der amerikanischen Nachrichtenmedien, den das „Project for Excellence in Journalism“ vorgelegt hat, wurden 2009 US-weit 5900 Redaktionsstellen in den Medien gestrichen, seit 2001 ist damit ein Drittel des damaligen Bestands verschwunden. Selbst Qualitätsblätter stellen ihre Berichterstattung vom teuren Recherche- auf den preiswerteren Meinungsjournalismus um (vgl. Piper 2010).

3 Nach Angaben des BDZV folgte einem Höchststand an Redakteursstellen von 15300 im Jahre 2000 ein Abbau von bislang gut 1000 Arbeitsplätzen (vgl. Röper 2010: 233, FN 9).

4 Eine aktuelle Übersicht liefern Weichert/Kamp 2009. 
sichtigung von Art. 5 GG realisiert werden kann. Hierfür wird auf die institutionenökonomischen Arbeiten der Forschergruppe um E. Ostrom zur Selbstorganisation von Commons zurückgegriffen, denn Professionen zählen in der Institutionenökonomik zu den „associational commons“ (4.).

Auf der entwickelten Argumentation aufbauend wird ein öffentlicher Finanzierungsmodus von Journalismus skizziert. Zur Begründung und Legitimierung einer öffentlichen Finanzierung des professionellen Journalismus wird wiederum die Theorie der Commons herangezogen. Für die Rezipienten stellt die journalistische Dienstleistung einen gemeinsam nutzbaren Ressourcenpool zur Wahrnehmung ihrer Rolle als Bürger dar, der hier in die Gruppe der „libertarian commons“ fällt, also Kollektivgutcharakter hat (5.).

Abschließend wird kurz erläutert, dass Journalismusförderung auch Medienförderung bedeutet, sofern und soweit das Medium professionellen Journalismus als zentrale Ressource nutzt (6.). Es folgt der Versuch einer Zusammenfassung (7.).

\section{Journalismus als gesellschaftliche Institution}

Journalismus wird in der modernen Gesellschaft nur noch im Zusammenhang mit Medienöffentlichkeit gedacht. Öffentlichkeit im allgemeinsten Verständnis ist ein im Prinzip frei zugängliches Kommunikationsforum, in dem Sprecher etwas mitteilen und Rezipienten diese Mitteilungen wahrnehmen, wobei immer mitschwingt, dass es sich um öffentliche, also kollektiv relevante Themen und Meinungen handelt, die dort besprochen und verhandelt werden. Peters (2007: 59) spricht von „Öffentlichkeit im emphatischen Sinn“, die durch Kommunikation unter Akteuren gebildet wird, „die aus ihren privaten Lebenskreisen heraustreten, um sich über Angelegenheiten von allgemeinem Interesse zu verständigen“.

Im Gegensatz zur Encounteröffentlichkeit (spontane interpersonale Kommunikation überschaubarer Gruppen) und Versammlungs- bzw. Themenöffentlichkeit (Rollendifferenzierung Sprecher/Hörer bei meist nicht mehr spontan ausgehandelten Themen) (vgl. Beck 2007: 105f.) verlieren Sprecher und Publikum bei Medienöffentlichkeit ihren interaktiven Zusammenhang. Als dritte Akteurgruppe tritt die der Vermittler auf und Öffentlichkeit weitet sich auf die Gesellschaft insgesamt aus (Neidhardt 1994: 101). Als zentrale, demokratietheoretisch die zentrale Gruppe von Vermittlern oder „Mediatoren" gelten Journalisten.

Journalismus wird, von der Mehrheit der Journalisten wie der Kommunikationswissenschaftler, heute als gesellschaftliche Dienstleistung verstanden (vgl. Donsbach 2008), die als unabhängige Orientierungsleistung über das gesellschaftliche Geschehen (Arnold 2009: 477) dem Bürger die Meinungsbildung und so das Funktionieren von Demokratie ermöglichen soll. Neben dieser sehr allgemeinen Definition gibt es in der Kommunikationswissenschaft zahlreiche theoretisch-konzeptionelle Annäherungen an den Journalismus, die im Ergebnis aber zu wenig begrifflicher Schärfe führen (vgl. Kepplinger 2004: 91, Neverla 1998: 61). Dennoch herrscht offenbar gesellschaftlicher Konsens über die genauere Spezifizierung der gesellschaftlichen Dienstleistung des Journalismus, wie Haller (2004: 133ff.) mit Hinweis auf empirische Daten zum Rollenselbstverständnis der Journalisten, zu Funktionszuweisungen an den Journalismus durch die Bevölkerung und das damit übereinstimmende Mediennutzungsverhalten darlegt. Danach herrscht Kon- 
sens zwischen den Kommunikationspartnern, dass Funktion des Journalismus ist bzw. sein soll, „dass

- gesellschaftliche Selbstverständigung als infiniter Kommunikationsprozess in Gang kommt bzw. bleibt;

- sich diese gesellschaftliche Verständigung an Aussagen über die (intersubjektiv erfahrbare) Ereignisrealität orientiert - und nicht etwa an realitätsfremden (fiktionalen) Selbst- und Fremdbildern oder Ideologien;

- aktuelle Vorgänge in der Ereignisrealität in Bezug auf (möglichst allgemein akzeptierte) Vorstellungen über das politische, wirtschaftliche und soziale Zusammenleben geprüft und bewertet werden“" (ebenda: 136).

Man kann sich dem Journalismus als gesellschaftlichem Phänomen nun auch mit institutionentheoretischen Ansätzen nähern (vgl. dazu näher Kiefer 2010). Das ist hilfreich vor allem, wenn man eine konsentierte Funktionszuweisung, wie die oben umschriebene, zu erklären und zu begründen versucht. Denn dass „Journalismus über den gesellschaftlich konsentierten Primat (verfügt), Themen zur öffentlichen Kommunikation bereitzustellen“, wie Rühl (1980: 329) kurz und prägnant feststellt, ist ja, auch wenn in der Kommunikationswissenschaft wohl unbestritten, nicht selbsterklärend und selbstbegründend.

Nun gibt es genauso wenig die Institutionentheorie, wie es die Journalismustheorie gibt, und selbst der Begriff der Institution bleibt wie der des Journalismus weitgehend vage. Eine vergleichsweise umfassende Definition, was unter einer Institution zu verstehen sei, die alle in den verschiedenen institutionentheoretischen Ansätzen weitgehend übereinstimmend als relevant betrachteten Kriterien aufgreift, bietet Göbel (2002: 3). Danach sind Institutionen:

- „Systeme von verhaltenssteuernden Regeln bzw. durch diese gesteuerte Handlungssysteme,

- die Probleme menschlicher Interaktion gemäß einer Leitidee ordnen,

- die für längere Zeit und einen größeren Kreis von Menschen gelten

- und deren Beachtung auf unterschiedliche Art und Weise durchgesetzt wird“.

Journalismus als Institution wäre danach

- ein durch Verhaltensregeln gesteuertes Handlungssystem (auf der Produktionsseite von Journalismus kann man an die Nachrichtenfaktoren oder journalistische Berufskodices denken, auf der Rezeptionsseite an Mediennutzungsgewohnheiten),

- das Problembereiche menschlicher Interaktion (hier öffentliche Kommunikation über gesellschaftlich relevante Themen) gemäß einer Leitidee (wie „Objektivität“ und „Vielfalt") ordnen soll,

- das nicht zuletzt durch die Verknüpfung mit Medien als Organisationen auf Dauer gestellt ist und auf der Produktions- wie Rezeptionsseite von Journalismus für einen großen Kreis von Menschen gilt,

- dessen Verhalten steuernde Regeln auf unterschiedliche Art und Weise durchgesetzt werden, auf der Produktionsseite z. B. durch Presserat oder Reputationsverlust bei Verstoß gegen Berufskodices, auf der Rezeptionsseite z. B. durch die Gefahr des Nicht-Mitreden-Könnens bei mangelnder Informiertheit.

Journalismus kann also im Sinne der Definition von Göbel als Institution begriffen werden. Die oben beschriebene Funktionszuweisung ist damit allerdings noch nicht voll erklär- und vor allem begründbar. Aussagen über intersubjektiv erfahrbare Ereignisrealitäten können ja allenfalls als Sollaufgabe dem Journalismus vorgegeben werden, an deren Erfüllung man glaubt oder nicht. Wie ist es also möglich, dass wir als Rezipienten journalistische Aussagen in der Regel als Realitätsbeschreibung akzeptieren, dass wir, 
wie Luhmann (1996: 6) sicherlich nur leicht übertreibend schreibt, das, „(w)as wir über unsere Gesellschaft, ja über die Welt, in der wir leben, wissen“, durch die Massenmedien wissen, dass die journalistischen und medial vermittelten Weltbilder „zur Synchronisation der Weltgesellschaft" (Bentele u. a. 2006: 115) beitragen?

Nach Searle (2005) müssen Institutionen, zumindest bestimmte Institutionen wie z. B. Geld, um als Institutionen „gemeinsames Wissen“ (Ostrom 1999: 66) der Gesellschaftsmitglieder, „institutionelle, Weltbilder”“ (Priddat 1996: 31) repräsentieren und so gesellschaftliche Kooperation ermöglichen zu können, eine Statusfunktion nach der

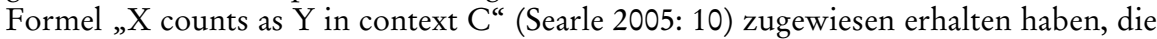
kollektiv anerkannt und akzeptiert ist. Geld z. B., so Searle, kann seine gesellschaftliche Funktion als allgemeines Zahlungsmittel ja nicht dank physischer Eigenschaften, sondern nur durch die kollektive Zuweisung einer entsprechenden Statusfunktion übernehmen. Journalismus scheint eine ähnliche Institution zu sein, die die oben beschriebene Ermöglichung gesellschaftlicher Selbstverständigung über kollektiv zu erreichende Ziele des Gemeinwesens nur dank der konsentierten Zuweisung und Akzeptanz einer entsprechenden Statusfunktion leisten kann.

Der normative Kern dieser Statusfunktion im Kontext einer Demokratie ist die Ermöglichung und Sicherung des für Demokratien zentralen Prinzips der Volkssouveränität. In einer so hoch differenzierten, komplexen, arbeitsteiligen und pluralistischen Gesellschaft, wie sie die modernen Massendemokratien darstellen, soll Journalismus mit seinen Wirklichkeitsbeschreibungen die Aufmerksamkeit des Einzelnen als Rezipient auf das kollektiv Relevante und Erforderliche lenken, das in einer von Werbung und PR durchtränkten, vorwiegend Unterhaltungsöffentlichkeit (vgl. Saxer 2007) ja keineswegs mehr für jedermann offensichtlich ist, und so die notwendige Informiertheit und die erforderlichen Lernprozesse der Staatsbürger mit Blick auf ihr Gemeinwesen ermöglichen. Es ist genau diese auf Interdependenz (Kohring 2004), auf die Ermöglichung gesellschaftlicher Kooperation gerichtete spezifische Selektivität, die konstitutiv ist für den Journalismus als Institution. Der journalistische Akteur übernimmt dabei eine soziale Agentschaft für den Bürger (vgl. Meyer/Jepperson 2005). Dieser institutionalisierte Agentenstatus ist die Basis für Vertrauen in das journalistische Handeln und für dessen Legitimation. Von daher gelten die institutionalisierten Wirklichkeitsbeschreibungen des Journalismus als wirklichkeitsgerecht.

Journalismus hat also eine spezifische, medienunabhängige, kollektiv zugewiesene Statusfunktion, die seinen Status als Institution auch in der Definition von Searle begründet. In der modernen Massendemokratie ist diese Statusfunktion aber nur mehr in der und durch die Sphäre der Öffentlichkeit zu realisieren, die wiederum vor allem die journalistischen Medien durch die Vervielfältigung journalistischer Aussagen und die Produktion von Publikum dafür herstellen. Auch wenn, wie Altmeppen (2006: 13) beklagt, „zwischen Medien und Journalismus häufig kein Unterschied gemacht“ werde, dürfen beide nicht gleichgesetzt werden, darüber besteht in der Kommunikationswissenschaft eigentlich auch Konsens (vgl. u. a. Kohring 2004: 160).

Medien haben, dem Metatrend zur Organisationsgesellschaft (Jäger/Schimank 2005; Türk et al. 2006) seit dem 19. Jahrhundert folgend, die Organisation des Journalismus (zentral: die Innovation der Redaktion) und als Wirtschaftsunternehmen vor allem auch dessen Finanzierung übernommen und seine Institutionalisierung in der Massendemokratie durch die Schaffung von gesellschaftsweiter Öffentlichkeit so erst ermöglicht. Zumindest die journalistischen Medien sind durch ihre Verknüpfung mit dem Journalismus dabei selbst zu Institutionen geworden. Journalismus ist in diesem institutionellen Arrangement allerdings die fundamentalere Institution, die das institutionelle Regelsys- 
tem und das institutionelle Feld bestimmt, in dem sich journalistische Medien bewegen. Sind journalistische Medien privatwirtschaftlich organisiert, ist Journalismus für sie zugleich die zentrale Ressource, die sie ökonomisch nutzen.

Die Überlagerung heute des Journalismus in der öffentlichen Wahrnehmung durch die Medien, die ihre gesellschaftlich herausgehobene Position und ihre verfassungsrechtlich geschützten Freiheitsspielräume dieser, so kann man wohl sagen, „unterschätzten Institution“ verdanken, mag viele Ursachen haben. Eine zentrale ist zweifellos der privilegierte Zugang der Medien zur Öffentlichkeit, die Gatekeeper-Position, die sie nicht nur für alle Sprecher, die sich an die Öffentlichkeit wenden wollen, einschließlich Werbewirtschaft, sondern auch für den Journalismus selbst haben oder zumindest bislang hatten.

Will man die einleitend gestellte Frage, ob Journalismus und journalistische Medien für die demokratische Gesellschaft unverzichtbar sind, beantworten, muss die Antwort für den Journalismus, den obigen Ausführungen folgend, ohne Einschränkung „ja “ lauten. Medien sind unverzichtbar, soweit und solange sie dem Journalismus die benötigte Öffentlichkeit zu schaffen vermögen; technische Struktur und Ausstattung der Medien, die ja dem jeweiligen Stand der Technik einer Gesellschaft und der Annahme dieser Techniken durch die Rezipienten folgen, scheinen dabei aber eher sekundär zu sein.

\section{Profession als autonome Kollegialorganisation}

Geht man, wie oben dargelegt, davon aus, dass Journalismus die demokratietheoretisch zentrale Institution im institutionellen Arrangement der Medien ist, muss eine öffentliche Finanzierung beim Journalismus und nicht bei den Medien ansetzen. Damit stellt sich die Frage nach einer Form sozialer Organisation des Journalismus, an die kommunikationspolitisches Handeln anknüpfen kann. Als Lösung wird hier das Konzept der Profession als einer formalen Institution vorgeschlagen.

Ein Rekurs auf das Konzept der Profession bietet sich grundsätzlich schon deswegen an, weil es sich dabei um ein dem Searle'schen Institutionenbegriff folgendes Konstrukt handelt: „X counts as Y in context C“ bzw. „Eine Profession kann eine Profession genannt werden, weil wir sie so nennen (ernennen und entsprechend handeln)" (Ortmann 2005: 286). ${ }^{5}$

Allerdings müssen für diesen performativen Akt der Professionsbildung einige Rahmenbedingungen vorliegen. Mieg (2003: 15ff.) nennt als wesentliche:

- Es gibt einen gesellschaftlich relevanten Problembereich und einen zugehörigen Bereich an Handlungs- und Erklärungswissen.

- Es gibt einen Bezug zu einem gesellschaftlichen Zentralwert.

- Es gibt eine weitgehend akademisierte Ausbildung.

- Es gibt einen Berufsverband bzw. eine berufsständische Vertretung.

Diese Rahmenbedingungen treffen auf den Journalismus zu. Die von Haller herausgearbeitete Funktion des Journalismus benennt den gesellschaftlichen Problembereich: die Ermöglichung gesellschaftlicher Selbstverständigung über die kollektiv relevante Ereignisrealität. Das journalistische Handlungs- und Erklärungswissen liefert heute die überwiegend akademische Ausbildung. Der gesellschaftliche Zentralwert, um den es hier geht, ist in der demokratischen Gesellschaft die Ermöglichung und Sicherung von Volkssouveränität.

5 Neverla (1998) zeigt zudem, wie sich im Konzept der Profession system- und handlungstheoretische Komponenten, zwischen denen die Theoriebildung der Journalistik und Kommunikationswissenschaft schwankt, aufeinander beziehen lassen. 
Als zentrale Kriterien von Professionen gelten deren Autonomie und Monopol in der Verwaltung des institutionell zugewiesenen Problem- und hochspezialisierten Wissensbereichs. Autonomie und Monopol betreffen die Kontrolle der Ausbildung und des Marktzutritts, die Macht über Definition und Bewertung der professionellen Leistungen, die Selbstbestimmung und Selbstkontrolle ihrer Arbeit durch die Professionals. „Professionen sind Berufe, die keiner anderen Berufsgruppe unterstellt sind“ (Klatetzki 2005: 277). Freidson (2001) spricht von der „Third Logic“, die professionelles Handeln kennzeichne in Abgrenzung zu der Logik des Marktes (Ideologie des „consumerism“) und derjenigen der Bürokratie (Ideologie des „managerialism“). Die professionelle Ideologie kennzeichnen „commitment to the quality of work“ (ebd.: 200), „its attachment to a transcendent value that gives it meaning and justifies its independence“ (221), „the professional's claim of license to balance the public good against the needs and demands of the immediate clients or employers" (222). Diese andere Logik und Ideologie ist für ihn „the soul of professionalism“ und sie lässt sich ohne Abstriche auf eine idealtypische Konzeption von Journalismus übertragen.

Auch wenn an dieser Stelle kein Überblick über den Stand der professionssoziologischen Diskussion angestrebt wird, sei noch auf zwei Aspekte mit Relevanz für den Journalismus hingewiesen: die Struktur professionellen Handelns und den Aspekt der Professionalisierungsbedürftigkeit.

Professionelles Handeln beruht auf komplexem abstraktem Wissen und gilt als nicht oder nur begrenzt routinisierbar. Strukturell setzt sich dieses Handeln nach Abbott (1988) aus drei Tätigkeiten zusammen: Diagnose, Inferenz und Behandlung (vgl. Klatetzki 2005: 262ff.). Durch die „Diagnose“ werden Informationen in das professionelle Wissenssystem aufgenommen, was die kulturelle Kompetenz des Professionellen voraussetzt, Realitäten wahrzunehmen, die der Laie nicht erkennen kann. Durch die „Behandlung" werden Informationen aus dem professionellen Wissenssystem nach außen übertragen. „Inferenz“ meint das kognitive Schlussfolgern und die Abklärung der zumeist mehrdeutigen Beziehung zwischen „Diagnose“ und „Behandlung“ mittels professioneller Kompetenz und bildet den Kern professionellen Handelns. Klatetzki (ebd.: 268) fasst zusammen, „dass professionelle Arbeit eine Form der Wahrnehmung bzw. Deutung von Wirklichkeit (Diagnose) und im Kern eine besondere Art schlussfolgernden Denkens ist (Inferenz). Die Waffen der Professionellen sind Ideen. Sie üben Kontrolle durch die Definition von Realität aus (..). Auf dieser Basis ist professionelle Arbeit zudem ein Bewirken von Wirkungen (Behandlung)“. Mit dieser Zusammenfassung lässt sich unbestreitbar auch journalistisches Handeln angemessen beschreiben.

„Professionalisierungsbedürftigkeit" wird vor allem in strukturfunktionalistischen und systemtheoretischen Ansätzen der Professionssoziologie primär von der Anbieterseite her gedacht. Kühl (2006: 19) schlägt vor, den Begriff auch von der Nachfrageseite her zu rekonstruieren, wenn dort „nach Surrogaten für fehlende Professionalität gesucht wird“. Solche Surrogate für den Rezipienten mögen bislang Medien gewesen sein („Qualitätszeitung“, „Leitmedium“ etc.), die in Zeiten des Medienumbruchs und des „Bürgerjournalismus“ allerdings an Eindeutigkeit verlieren. Will man eine nachfrageseitige Professionalisierungsbedürftigkeit des Journalismus rekonstruieren, ist ein Verweis von Ortmann (2005: 295) auf den Beitrag, den die Institutionenökonomik (Stichworte: Vertrauensgüter, asymmetrische Information, Reputation, signalling, credible commitments etc.) hier für die Professionssoziologie leisten könne, von besonderem Interesse, weil in der Medienökonomik bereits Lehrbuch-Standard (vgl. u. a. Heinrich 2001: 98ff., Kiefer 2005: 332ff.). 
Freidson (1983: 19ff.) unterscheidet wie viele andere Professionssoziologen zwischen zwei Professionskonzepten: dem weiteren ausbildungsorientierten, das von weitgehend individuell definierten Formen professionellen Handelns ausgeht, und dem engeren, das an institutionelle und ideologische Merkmale der Profession zur Abgrenzung von anderen Berufen anknüpft. Journalismus galt bislang als Profession im weiteren Sinn (vgl. Mieg 2003: 13). Voraussetzung für eine kollektive Finanzierung, so die hier vertretene These, ist die Begründung und Überführung in eine Profession gemäß dem engeren Konzept. Das setzt vor allem eines voraus: eine Bestimmung, wer zur Profession gehört, und eine soziale Schließung (vgl. Feidson 2001: 199ff.). Beides wiederum setzt die Beantwortung der schon 1998 von Neverla gestellten Frage voraus: „Welche ,Journalismen” wollen wir ernsthaft zum Journalismus zählen?" (ebd.: 60). Eine entsprechende Definitions- und Abgrenzungsarbeit, deren Schwierigkeit beachtlich ist und die hier auch nicht ansatzweise versucht werden soll, müsste im Rahmen eines Professionsbildungsprojekts von Journalismuspraktikern und -theoretikern geleistet werden. Darüber hinaus stellt sich die Frage: Wie bildet man eine Profession? Hier kann die institutionenökonomische Theorie der Commons Hilfestellung leisten, die Professionen als eine Form von Commons begreift.

\section{Selbstorganisation in Anlehnung an die Theorie der Commons}

E. Ostrom (1999, 2005), Wirtschaftsnobelpreisträgerin 2009, hat mit ihren langjährigen theoretischen und empirischen Arbeiten zur Organisation von Allmenden, also von gemeinsam nutzbaren Ressourcen oder Commons eine in der Ökonomik bislang vorherrschende pessimistische Annahme in Frage gestellt, nämlich dass rational ihr Eigeninteresse verfolgende Mitglieder einer Gruppe nicht in der Lage seien, ein gemeinsames Gruppenziel zu erreichen. Metaphern wie die von der „Tragik der Allmende“, aber auch die von Olson entwickelte „Logik des kollektiven Handelns“ (Olson 1992), ebenso die in der Ökonomik gern benutzte spieltheoretische Figur des „Gefangenendilemmas“: Sie verweisen alle auf diese pessimistische Annahme.

Von Allmenderessourcen oder Commons wird in der Regel im Zusammenhang mit natürlichen Ressourcen wie Fischgründen, Wasserreservoirs oder Weiden gesprochen, die für viele nutzbar sind, bei deren rücksichtslosem Umgang damit allerdings die Gefahr ihrer dauerhaften Schädigung besteht (die Diskussion der allgegenwärtigen Umweltprobleme dreht sich um genau diese Erscheinungen). Heute ist die Tragik der Allmende eine ökonomische Metapher, die auf eine Vielzahl von Problemen kollektiven Handelns auch außerhalb natürlicher Ressourcennutzung Anwendung findet (vgl. Ostrom 1999: 4). Bislang sahen Ökonomen wie Politologen für die Lösung solcher Probleme nur zwei Wege: Privatisierung oder staatliche Regulierung. Ostrom verweist nun auf eine dritte Möglichkeit: die Selbstorganisation und Selbstverwaltung. Individuen in einer Situation strategischer Interdependenz entwickeln selbst die Regeln, die es ermöglichen, die dilemmatische Struktur aufzulösen, verpflichten sich auf diese Regeln und überwachen deren Einhaltung.

Ostroms Arbeit könnte wegleitend sein für einen Professionsbildungsprozess des Journalismus, denn, wie erwähnt, institutionenökonomisch gelten Professionen als Commons, genauer als „associational commons“ (Levine 2007: 250f.). „Associational commons“ meint, dass, im Gegensatz zu den unter 5. zu diskutierenden „libertarian commons“, eine definierte Gruppe ein Ressourcensystem nutzt. Im Falle der Profession oder des Journalismus ist dies ein intangibles Ressourcensystem, denn ihre „Allmen-

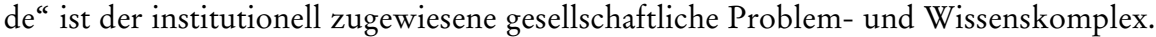
Ostrom hat einen Katalog von „Design Principles“ für eine erfolgreiche Selbstorgani- 
sation von Commons (Ostrom 2005: 258ff.) erarbeitet, der als Katalog von Erfordernissen, die bei einem Professionsbildungsprozess von Journalismus zu beachten sind, gelesen werden kann.

Das erste und wichtigste der Erfordernisse ist: „Clearly defined boundaries“. Das Ressourcensystem, seine Grenzen, der Personenkreis der berechtigten Nutzer müssen klar definiert und festgelegt werden. Für Ostrom ist die Zugangsbeschränkung auf Personen, die den vereinbarten Regeln zustimmen, der wichtigste Schritt. Zu wissen „who is in and who is out of a defined set of relationships" ist die Voraussetzung für Kooperationsbereitschaft, Vertrauen, Reziprozität und Verantwortung (ebd.: 261). Grenzziehungen via Definition und soziale Schließung sind für Ostrom also ebenso ein unabdingbares Organisationserfordernis, wie sie bei der Profession als zentral erachtet werden. Die gerade angesprochene Definitions- und Abgrenzungsarbeit ist also unerlässlich.

Zentrales Erfordernis ist weiter: „Collective-choice arrangements“. Die AllmendeNutzer selbst handeln das für sie gültige Regelsystem und später allfällige Regeländerungen aus. Autonomie ist das professionssoziologische Stichwort. Zu dem Regelsystem gehören unabdingbar:

- „Monitoring“. Vertrauen und Reziprozität sind wichtig, aber nur selten ausreichend. Durch ein internes Monitoring-System soll das regelgerechte Verhalten der Mitglieder kontrolliert, aber auch der Zustand der Ressource beobachtet werden.

- „Graduated sanctions“. Ein internes Sanktionssystem bei Regelverstößen soll berücksichtigen, dass ,jeder einmal einen Fehler machen kann“, aber wiederholten Regelbruch soll es wirksam verhindern.

- Durch die Installation eines Monitoring- und Sanktionssystem wird berücksichtigt, dass selbstorganisierende Regimes stark auf „quasi-voluntary“ Kooperation bauen, weniger auf Zwang, aber auch nicht auf reine Freiwilligkeit.

- "Conflict-resolution mechanisms“. Konflikte, die immer entstehen können, wenn Regeln interpretiert werden, müssen schnell und kostengünstig in internen Arenen gelöst werden.

Monitoring, Sanktion und Konfliktlösung sind bei der klassischen Profession in der Regel bei der Kammer angesiedelt. Eine vergleichbare formale Organisation für den Journalismus müsste also geschaffen werden. ${ }^{6}$ Die Beobachtung und auch Weiterentwicklung des Ressourcensystems, also des dem Journalismus institutionell zugewiesenen gesellschaftlichen Problem- und Wissensbereichs, ist vor allem Sache der einschlägigen Wissenschaft, hier speziell Journalistik und Kommunikationswissenschaft, die ja zur Profession zählen (vgl. Rühl 2004: 128).

Für den Journalismus ist noch eine weitere von Ostrom herausgearbeitete Voraussetzung für eine robuste Selbstorganisation zentral: die Anerkennung des Selbstorganisationsrechts der Allmendenutzer durch den Staat. Zwar sind Professionen ohne Staat nicht denkbar, denn eine übergeordnete gesellschaftliche Instanz, die über entsprechende Macht verfügt, muss ihr Monopol garantieren (vgl. Littek et al. 2005: 75). Und Professionen sind „(i)m Gegensatz zur ihrer Kennzeichnung als ,Freie“ Berufe (...) hochgradig regulierte Berufe“ (ebd.: 77). In Deutschland dominiert staatliche Regulierung als eine der Berufsausübung vorgelagerte Regulierung, die vor allem der Qualitätssicherung dient (vgl. ebd.: 109), die sich mit Blick auf Art. 5 GG hier aber weitgehend auf die Ermöglichung der Selbstorganisation von Journalismus als Profession und die Sicherung seiner Finanzierung beschränken müsste.

6 Zur mangelnden Eignung des Presserats in seiner derzeitigen Form vgl. z. B. die Kritik von Pöttker 2003. 
Eine öffentliche Finanzierung des Journalismus setzt also zunächst einmal voraus, dass ein Prozess in Richtung Professionsbildung unter Berücksichtigung der von Ostrom erarbeiteten „Design principles“ eingeleitet wird. Das kann nicht vom Journalismus allein in Gang gesetzt werden, sondern erfordert gesellschaftliche, vor allem wissenschaftliche, politische und gesetzgeberische Initiativen. Der eher düstere Ausblick auf die nähere und fernere Zukunft des Journalismus schafft dafür insofern günstige Ausgangsbedingungen, als über den Erhalt und die Weiterentwicklung der Institution Journalismus nicht nur wirtschaftlich, sondern in Orientierung an ihrer Leitidee und Statusfunktion in Demokratien reflektiert werden muss. Der Gesellschaft, die auf diese Institution nicht verzichten will, bleibt angesichts der gebotenen Staatsferne einerseits und den Überwucherungsgefahren durch ökonomische Rationalität bei Einbettung in die Privatwirtschaft andererseits wenig anderes übrig, als auf Erhalt durch Selbstorganisation zu bauen. Dass dies nicht ohne Risiko ist, muss gesehen werden. Der Begriff der „Commons“ ist nicht wertgeladen - „its outcome can be good or bad, sustainable or not" (Hess/Ostrom 2007: 14). Aber die Wahrscheinlichkeit scheint groß, dass bei Beachtung der Befunde von Ostrom für eine Organisation des Journalismus nach dem Konzept der klassischen Profession die Journalisten, gerade auch als rationale Akteure und Homines Oeconomici (Fengler/Ruß-Mohl 2005), ihre „Allmende“, die ja Basis ihrer wirtschaftlichen Existenz ist, gemäß den via Ausbildung vermittelten professionellen Regeln und Verpflichtungen pflegen, wobei die gewonnene Reputation des Berufstandes die quasi-freiwillige Kooperation zusätzlich abstützen dürfte. ${ }^{7}$

\section{Umrisse eines Modus öffentlicher Finanzierung von Journalismus}

In Deutschland wie in anderen europäischen Ländern gibt es bereits ein Modell der kollektiven Finanzierung von Journalismus und journalistischen Medien: die öffentlichrechtliche Organisationsform ${ }^{8}$. Einer Übertragung dieses Modells auf die Zeitung als Prototyp journalistischer Medien soll hier allerdings nicht das Wort geredet werden: Dies erscheint - für eine Zeitungslandschaft - wenig praktikabel, und der Wettbewerb zumindest zwischen öffentlich-rechtlichem Rundfunk und privatwirtschaftlich organisierter Tageszeitung scheint im Rückblick auf die Entwicklung Deutschlands zur Demokratie durchaus positive Effekte ausgelöst zu haben. Die privatwirtschaftliche Organisationsform von Medien, ob kommerziell oder z. B. genossenschaftlich organisiert, bleibt hier also unberührt, soll für journalistische Medien durch ein mit der Förderung verbundenes wirtschaftliches Anreizsystem aber effektiver mit Blick auf die Funktion des Journalismus werden.

Ausgehend von dem bislang Dargelegten wird hier ein Finanzierungsmodus vorgeschlagen, der mit einem Professionsbildungs- und Selbstorganisationsprozess des Journalismus zeitlich und inhaltlich verknüpft ist.

7 Die teils skeptische Beurteilung der Zukunft von Professionen (vgl. z. B. im Sammelband von Klatetzki/Tacke (2005) die Beiträge von Stichweh, Tacke, Klatetzki) könnte dabei durchaus als Anregung dienen, welche „Klippen“ im Professionsbildungsprozess mit bedacht werden müssen.

8 Inwieweit das hier vorgeschlagene Modell Rückwirkungen auch auf die öffentlich-rechtliche Medienorganisationsform haben könnte, bleibt hier unerörtert. Beide Modelle folgen aber wohl grundsätzlich unterschiedlichen Ordnungsvorstellungen, deren - auch rechtliche - Kompatibilitäten erst näher geprüft werden müssten. 
Eine öffentliche Finanzierung von Journalismus wäre danach an Bedingungen zu binden, die wie folgt aussehen könnten:

1. Finanziell gefördert werden Redaktionen, Redaktionsbüros und vergleichbare Einrichtungen ${ }^{9}$, die mit Blick auf die Inhalteproduktion ausschließlich und autonom von Journalisten geleitet werden.

2. Journalist ist eine geschützte, an theoretische und praktische Ausbildungsgänge mit entsprechenden Examina gebundene Berufsbezeichnung.

3. Journalismustheoretiker und -praktiker entwickeln in Zusammenarbeit mit Vertretern der Medien- und Bildungspolitik sowie Medienrechtlern ein Ausbildungs- und Prüfungscurriculum, dessen erfolgreiche Absolvierung den Zugang zum Beruf des Journalismus eröffnet. Dabei ist auf Abgrenzungen zu anderen Kommunikationsberufen, etwa im Feld der Public Relations, der Werbung und der Unterhaltung, besonders zu achten. ${ }^{10}$

4. Das Ausbildungsverfahren sollte ähnlich wie bei Pädagogik, Jura oder Medizin zweigegliedert sein in ein Studium an der Universität und eine eher praxisorientierte, „tacit-Wissen“ vermittelnde „Referendar“-Zeit. Jede der Stufen schließt mit einem Examen. Die Berufsbezeichnung Journalist setzt beide Examina voraus.

5. Die „Referendar“-Ausbildung erfolgt ausschließlich in Redaktionen, Redaktionsbüros und vergleichbaren Einrichtungen, die als förderungswürdig anerkannt sind. Abschluss dieser Ausbildungsphase ist eine externe Prüfung. Über das Prüfungsgremium (z. B. eine Kammer) ist im Rahmen der Festlegung des Ausbildungscurriculums zu befinden.

Öffentliche Förderung setzt damit nicht an der Qualität der Produkte, mit den bekannten inhaltlichen und messtechnischen Problemen an, sondern an der formalisierten Professionszugehörigkeit des Personals.

Die Vorstellung, dass Informations- und Meinungsbildungsfreiheit eine unbedingte, d. h. an keine Qualifikationsnachweise gebundene Berufszugangsfreiheit zum Journalismus erfordere, wird aufgegeben. Wenn, wie Rühl (2004: 128f.) schreibt, Journalismus immer „der Journalismus einer Gesellschaft im Wandel“ ist, dann ist es legitim und erforderlich, in einer sich wandelnden Gesellschaft auch neue Vorstellungen von seiner gesellschaftlichen Organisation zu entwickeln. Wie Donsbach u. a. (2009: 122ff.) in ihrer Studie zur öffentlichen Wahrnehmung des Journalismus ermittelten, befürworten 89 Prozent der deutschen Bürger eine verbindliche Ausbildung für Journalisten, ein deutlicher Hinweis auf eine Professionalisierungsbedürftigkeit aus Sicht der Rezipienten, was die Akzeptanz auch eines damit verknüpften Finanzierungsprogramms zweifellos erhöht. Die Befunde der Studie machen auch die durchaus differenziert wahrgenommene und grundsätzlich akzeptierte Bedeutung dieser gesellschaftlichen Institution aus Sicht der Bürger deutlich. Der gesellschaftliche Konsens über die Funktion des Journalismus, wie sie von Haller (2004) herausgearbeitet wurde, ist (auch) von Seiten des Publikums also keineswegs aufgekündigt. Für eine öffentliche Finanzierung ist dies eine wichtige Voraussetzung.

Die bisherige, überwiegend privatwirtschaftlich organisierte Finanzierung des Journalismus ist aus ökonomischer Sicht ein sehr spezielles Arrangement, dessen allokative Effizienz selbst klassische Ökonomen (vgl. u. a. Schröder 2008: 205ff.) in Frage stellen, vor allem weil die Selektion der Inhalte weniger der Wertschätzung des Publikums als

9 Die Förderung könnte grundsätzlich auch bei dem einzelnen Professionsmitglied ansetzen (und wird das bei Freiberuflichkeit auch tun müssen), die ja ebenfalls öffentlich zu finanzierenden Transaktionskosten werden dadurch aber erheblich erhöht.

10 Vgl. dazu auch Rühl (2008: 571ff.) und seine Einforderung einer Journalistikpolitik. 
den Zielgruppenpräferenzen der Werbewirtschaft folgt. Wenn Donsbach u. a. schreiben, dass „das Publikum weniger Boulevard und mehr sachliche Substanz verlange“ (ebd.: 131), dass aus Sicht vieler Bürger „das Nachrichtenangebot an der Nachfrage vorbei(gehe)" (ebf.: 132), dann sind die im Finanzierungsarrangement begründeten Ineffizienten angesprochen. Dabei ist die Medien- und Journalismusfinanzierung via Werbung für den Rezipienten keineswegs kostenlos. Seine Kostenbeteiligung in der Rolle als Konsument der beworbenen Produkte ist lediglich intransparent und gehorcht nicht dem Äquivalenzprinzip.

Wie aber lässt sich eine öffentliche Finanzierung des Journalismus institutionenökonomisch begründen? Auch die Information und das Wissen, die der Journalismus für die Gesellschaft produziert und bereitstellt, sind institutionenökonomisch Commons, ein Ressourcensystem für die Mitglieder der Gesellschaft in ihrer Rolle als Bürger. Es sind in der Unterscheidung von Levine (2007: 250f.) „libertarian commons“, denn jeder kann und darf sie nutzen, was ihren Kollektivgutcharakter unterstreicht. Eine Selbstorganisation der Bürger zur Verwaltung, Erhaltung und Finanzierung von „libertarian commons“ ist institutionenökonomisch unwahrscheinlich. Dafür ist die Gruppe zu groß: alle Bürger gehören zu dem berechtigten Nutzerkreis, Grenzziehungen in diesem Fall würden Art. 5 GG zudem massiv verletzen, und das Freerider- bzw. Trittbrettfahrerproblem (vgl. u. a. Kiefer 2005: 133) ist kaum zu überwinden. Außerdem wären die Kosten der Entscheidungsfindung und -durchsetzung ineffizient hoch. In einer solchen Situation ist die Delegation der Organisationsaufgabe an die Volksvertreter eine in einer repräsentativen Demokratie bewährte Lösung. Da es sich bei dem Ressourcensystem journalistische Information nicht, wie z. B. bei einer Weide, um ein ständig in bekannter Qualität nachwachsendes, sondern um ein immer wieder kostenintensiv neu zu produzierendes Ressourcensystem handelt, sind zentrale, von den Volksvertretern zu lösende Probleme:

1. die Voraussetzungen zu schaffen, dass diese fortwährende Neuproduktion erfolgt und so erfolgt, dass die Bürger daraus den erwarteten Nutzen ziehen können, und

2. die Finanzierung der kontinuierlichen Neuproduktion sicherzustellen.

Der hier entwickelte Lösungsvorschlag für das erste Problem ist die kollegiale Selbstorganisation des Journalismus gemäß dem Konzept der Profession unter Beachtung der von Ostrom entwickelten Organisationsvoraussetzungen. Den Lösungsvorschlag für das zweite Problem weiterzuentwickeln und zu begründen ist Ziel dieses Kapitels.

In den USA haben die technischen Möglichkeiten der Digitalisierung und des Internets eine Diskussion in der Wissenschaft dahingehend ausgelöst, ob für „KnowledgeCommons" Zugangsbarrieren, wie sie z. B. die Verwertungsrechte darstellen, noch angemessen sind (vgl. Hess/Ostrom 2007). Ein Argument, wissenschaftliche Abhandlungen tantiemenfrei allen Interessierten zugänglich zu machen, ist, dass der Autor für die vorausgehende wissenschaftliche Arbeit, auf der die Veröffentlichung basiert, von der Gesellschaft schon entgolten wurde. „Scholars can afford to donate their journal articles because they are paid salaries by universities“ (Suber 2007: 175). Diese „öffentliche“ Finanzierung mache den Autor zudem marktunabhängig in seiner Arbeit und erlaube akademische Freiheit. Man kann die Argumentation im Zusammenhang mit Journalismus und Journalismusfinanzierung nun genau umdrehen: Journalismus muss öffentlich finanziert werden, weil seine Leistungen als libertarian commons allen Gesellschaftsmitgliedern in ihrer Rolle als Bürger offenstehen und in einer Demokratie ohne finanzielle Barrieren offenstehen müssen. Journalismus als gesellschaftliche Institution erfordert ja beides, die Produktion und die Rezeption seiner „Dienstleistungen“, nur dann kann die institutionelle Funktion erfüllt werden. Hier stellt Journalismus im Übrigen 
auch keine Ausnahme dar. Information und Wissen sind, halten Ostrom/Hess (2007: 53) fest, ,a ,flow ressource' that must be passed from one individual to another to have any public value“. Das gilt für die journalistische Information in besonderem Maß, wobei die „Fließgeschwindigkeit“ hier besonders hoch ist.

Der Vorteil der Theorie von den Commons ist, hierin der „Third Logic“ des Konzepts der Profession nicht unähnlich, dass sie Ordnungsformen zwischen Markt und Staat bereitzustellen und zu begründen versucht. „The metapher of the commons provides a language to explain how the extraordinary public assets invested in the nation's information infrastructure can deliver democratic opportunities for the partizipation of all citizens. (...) The commons elevates individuals to the role above mere consumers in the marketplace, shifting the focus to their rights, needs, and responsibilities as citizens" (Kranich 2007: 93f.).

Öffentliche Finanzierung meint hier nicht, dass alles notwendige Geld aus dem allgemeinen Steuertopf kommen muss, sondern dass die Finanzierung des Journalismus vom Gesetzgeber als eine marktunabhängige organisiert und sichergestellt wird. Für mögliche Einnahmequellen bietet das erwähnte Papier von Downie/Schudson (2009) einiges Anregungspotenzial. Ergänzen lassen sich die Vorschläge um einen steuerlich differenzierten Umgang nicht nur mit Medien, sondern auch mit Werbung und PR. ${ }^{11}$ So lassen sich ja durchaus Modelle vorstellen, dass Werbeaufwendungen von Unternehmen, die in Medien ohne geförderte Redaktion fließen oder die Vermittlung von Medien überhaupt vermeiden, mit Blick auf ihre steuerliche Abzugsfähigkeit anders behandelt werden als Werbung, die zur Finanzierung journalistischer Medien und damit des Journalismus beiträgt. Auch ein steuerlicher Beitrag von PR zur Finanzierung des Journalismus ist begründbar, da sie sich dieser Ressource und des dadurch geschaffenen Vertrauenspotenzials ja gerne für die eigenen partikularen Anliegen zu bedienen versuchen. Auch eine steuerliche Differenzierung der Medien danach, ob sie eine geförderte Redaktion mit unterhalten oder nicht, lässt sich begründen. Die einen tragen zum Erhalt des Journalismus als kulturelle Institution bei, die anderen nicht. Das sind nur einige, den allgemeinen Steuerzahler kaum belastende Einnahmequellen, weitere lassen sich sicherlich finden. Stiftungen und Spenden sollten durch steuerliche Begünstigungen angeregt werden, den Rest, wenn alle anderen Quellen ausgeschöpft sind, muss die Allgemeinheit tragen. Es ist hier nicht die Absicht, einen detaillierten Finanzierungsplan von Journalismus vorzulegen, sondern es soll mit Beispielen lediglich die Möglichkeit als realistisch aufgezeigt werden, diese Finanzierung öffentlich zu organisieren. Muss diese Organisation der Finanzierung durch den Gesetzgeber geregelt werden, so ist die Verfügung über die Mittel und ihre konkrete Verteilung mit Blick auf die gebotene Staatsferne wohl bei den Organen der journalistischen Kollegialorganisation anzusiedeln.

\section{Medienförderung - nicht nur wirtschaftlich}

Die oben angedeuteten Beispiele für die Erschließung von Finanzierungsquellen machen bereits deutlich, dass Journalismusförderung auch Medienförderung bedeutet, sofern und soweit das jeweilige Medium dem autonomen professionellen Journalismus ein Forum bietet. Erreicht wird sowohl eine direkte Förderung (Entlastung bei den Aufwen-

11 Solche Maßnahmen lassen sich mit dem Äquivalenzprinzip begründen. So soll z. B. im Falle von PR für den Nutzen, den Gruppen mit partikularen Interessen aus der öffentlichen Dienstleistung Journalismus ziehen, indem sie dessen Aktivierungspotenzial von Aufmerksamkeit für die eigenen Belange einzuspannen versuchen, eine angemessene Kostenbeteiligung geleistet werden. 
dungen für die förderungswürdige Redaktion) als auch eine indirekte (steuerliche Begünstigungen von Medien mit förderungswürdiger Redaktion gegenüber solchen ohne), und beide Formen setzen für die Medien wirtschaftliche Anreize in Richtung des professionellen Journalismus. Begünstigt werden förderungswürdige journalistische Medien auch durch eine oben angesprochene unterschiedliche steuerliche Behandlung der Werbeaufwendungen. Auch hier zielen die Anreize, diesmal für die werbetreibende Wirtschaft, in Richtung Förderung des professionellen Journalismus: Neben den finanziellen Anreizen durch steuerliche Begünstigung dürfte auch der Distinktions- und wahrscheinliche Reputations- und Seriositätsgewinn geförderter Medien beim Publikum für die werbetreibende Wirtschaft von Interesse sein. Für das Publikum ist die skizzierte Journalismusfinanzierung ja zentrale Orientierungshilfe, bei welchen Medien professioneller Journalismus zu erwarten ist. Für eine Zeitung, die im Wettbewerb steht oder die auf ihre Online-Angebote aufmerksam machen möchte, ist die Förderung zweifellos ein Argument, das werblich genutzt werden kann.

Die Förderung bietet auch eine Basis für die Wiederbelebung einer Medienkritik. Nicht nur die finanzielle Unabhängigkeit des Journalismus, sondern insbesondere die Definition von Leistungskriterien professionellen Handelns ermöglichen und erfordern auch einen selbstreflexiv-kritischen Umgang mit dem eigenen Tun und dem der Mitspieler im institutionellen Feld. „Monitoring“ sollte angesichts der Zentralität der Institution Journalismus in demokratischen Gesellschaften nicht nur als internes Kontrollsystem verstanden werden, sondern auch als Transparenz- und Rechenschaftspflicht gegenüber der Gesellschaft. Ganz im Sinne der Theorie von den Commons würde dies eine Überwindung der Rolle des Publikums nur als Konsument von Medienprodukten begünstigen und sein Hineinschlüpfen in die Rolle des Bürgers erleichtern, der für sein Mediensystem und dessen Qualitäten mit verantwortlich ist. Für die Medien selbst muss das nicht von Nachteil sein. Wenn ihre Angebote, die ja heute zum großen Teil als Produkte einer Wegwerfgesellschaft konzipiert sind und so auch rezipiert werden, für das Publikum wieder wichtig werden, ist auch das eine Medienförderung, die den Medienorganisationen zumindest mittelfristig auch wirtschaftlich hilft.

Der hier skizzierte Modus einer öffentlichen Finanzierung des Journalismus soll zunächst medienunabhängig sicherstellen, dass diese für demokratische Gesellschaften zentrale Institution funktionsfähig bleibt. Dass mit der öffentlichen Finanzierung von Journalismus zahlreiche Formen der direkten und indirekten Förderung auch von journalistischen Medien, unabhängig davon, welcher Kanäle und technischen Möglichkeiten sie sich für die Schaffung medialer Öffentlichkeit bedienen, verbunden sind, wurde andeutungsweise skizziert und ist ein gewollter Nebeneffekt. Andere und weitere staatliche oder auch private Förderungsmaßnahmen z. B. zur Abstützung der Lesekultur oder einer visuellen Kultur in Film und Fernsehen sind davon nicht tangiert, sie sollten allerdings klar von einer Journalismusförderung durch öffentliche Finanzierung abgegrenzt werden.

\section{Zusammenfassung}

Das hier skizzierte Modell einer Journalismusfinanzierung folgt der berühmt-berüchtigten Suche nach dem dritten Weg: Eine Lösung wird nicht vom Markt erhofft - eine Strategie, welche die privatwirtschaftlichen Medienorganisationen in Form von PaidContent-Angeboten vor allem für Internet und Handy (vgl. Siebenhaar 2010) zu entwickeln und zu realisieren versuchen -, sie wird aber auch nicht als Übertragung des öffentlich-rechtlichen Organisationsmodells auf alle journalistischen Medien versucht. 
Zentral für die Suche nach dem dritten Weg ist die Trennung zwischen Journalismus und Medien. Sie ist theoretisch begründbar, wenn man Journalismus als eine eigene Institution begreift, die relativ zu den Medienorganisationen als die demokratietheoretisch eigentlich relevante einzustufen ist. Medienorganisationen waren bislang zentral für den Journalismus, weil sie dessen Organisation und Finanzierung in breitem Umfang übernommen hatten und so selbst institutionellen Charakter gewannen. Der technische und gesellschaftliche Wandel untergräbt nun ihr ohnehin ambivalentes Potenzial der Journalismusfinanzierung. Medien bleiben auch in dem hier skizzierten Finanzierungsmodell, zumindest auf absehbare Zeit, ein zentrales Organisationsgerüst für den Journalismus, in dessen Rahmen sich ein Großteil der journalistischen Arbeit vollzieht, und sie bleiben, wiederum zumindest auf absehbare Zeit, weiterhin die Produzenten von Publikum, Publikumsaufmerksamkeit und damit von Öffentlichkeit, die dem Journalismus eine Erfüllung seiner gesellschaftlich konsentierten Statusfunktion ermöglicht.

Das traditionelle institutionelle Arrangement von Journalismus und Medien ändert sich allerdings gravierend, die gegenseitige Abhängigkeit wird lockerer. Finanzielle Förderung des Journalismus wird an dessen Autonomie durch Professionsbildung und Selbstorganisation gebunden. Die notwendigen Schritte dorthin wurden skizziert. Begründet werden die Vorschläge mit der institutionenökonomischen Theorie der Commons und den empirischen Befunden dazu.

Angesichts der Zentralität der Institution Journalismus und der veränderten gesellschaftlichen, vor allem technisch-medialen Ausgangsbedingungen wird davon ausgegangen, dass der Staat eine Neuorganisation des Journalismus einschließlich seiner Finanzierung ermöglichen muss. Mit Blick auf die gebotene Staatsferne bedeutet dies, dass er die Rahmenbedingungen schafft und organisiert:

- für die mit dem Professionsbildungsprojekt verbundenen Aushandlungsprozesse über Professionszugehörigkeit, -qualifikation, -leistungen etc.;

- für die soziale Organisation des Journalismus als autonome Kollegialorganisation;

- für eine öffentliche Finanzierung, die dem Journalismus die autonome Produktion seiner gesellschaftlichen Dienstleistung ermöglicht.

Das Abenteuer für die Gesellschaft einer professionellen Selbstorganisation des Journalismus erscheint weniger groß, wenn man sich die bisherige Lösung des Problems vergegenwärtigt. Dieses Problem besteht ja bekanntlich darin, dass journalistische Dienstleistungen als öffentliche Güter zwar einer Finanzierung bedürfen, diese über den Markt als Refinanzierungsmechanismus jedoch nicht ausreichend erreicht werden kann. Die Querfinanzierung des Journalismus aus dem Werbemarkt, wie sie die privatwirtschaftlichen Medienorganisationen bislang organisierten, ermöglichte dessen Leistungen, machte die Institution aber gleichzeitig zu einer zentralen Ressource privater Wirtschaftsunternehmen, die sie als rationale wirtschaftliche Akteure gemäß ihren unternehmerischen Kalkülen einsetzen. Dass dies mit erheblichen Ineffizienzen auch aus ökonomischer Sicht verbunden ist, wurde hier diskutiert. Wie groß der Mangel an gesellschaftlicher Effektivität dieses Modells war und ist, darüber streitet die kommunikationswissenschaftliche Wirkungsforschung, ohne eine Antwort je geben zu können, auch, weil eine verbindliche Referenzgröße nicht vorliegt. Folgt man den theoretischen und empirischen Erkenntnissen der Institutionenökonomen wie den Befunden der Professionssoziologie, dann kann die Selbstorganisation eines professionellen, seiner gesellschaftlichen Aufgabe und Verantwortung bewussten Journalismus als das im Vergleich mit der bislang gepflegten Praxis gesellschaftlich risikoärmere Abenteuer bewertet werden. Es sollte als dritter Weg zwischen Markt und Staat also zumindest erprobt werden, zumal andere Lösungen, die Institution Journalismus möglichst unbeschadet in einer 
Welt des technischen, wirtschaftlichen und gesellschaftlichen Umbruchs zu erhalten, kaum sichtbar sind.

Dass diese hier vorgelegte erste und entsprechend grobe Skizze dieses Wegs noch sorgfältiger Ausarbeitung und Präzisierung bedarf, sei abschließend zumindest angemerkt.

\section{Literatur}

Abbott, Andrew (1988): The System of Professions: An Essay on the Devision of Expert Labour. Chicago.

Altmeppen, Klaus-Dieter (2006): Journalismus und Medien als Organisationen. Leistungen, Strukturen und Management. Wiesbaden.

Arnold, Klaus (2009): Qualitätsjournalismus. Die Zeitung und ihr Publikum. Konstanz.

Beck, Klaus (2007): Kommunikationswissenschaft. Konstanz.

Bentele, Günter, Hans-Bernd Brosius, Otfried Jarren (Hg.) (2006): Lexikon Kommunikations- und Medienwissenschaft. Wiesbaden.

Breunig, Christian (2005): Paid Content im Internet - ein erfolgreiches Geschäftsmodell? In: Media Perspektiven, Heft 8, 407-418.

Donsbach, Wolfgang (2008): Im Bermuda-Dreieck. Paradoxien im journalistischen Selbstverständnis. In: Pörksen, Bernhard, Wiebke Loosen, Armin Scholl (Hg.), Paradoxien des Journalismus. Theorie - Empirie - Praxis. Wiesbaden, 147-164.

Donsbach, Wolfgang, Mathias Rentsch, Anna-Maria Schielicke, Sandra Degen (2009): Entzauberung eines Berufs. Was die Deutschen vom Journalismus erwarten und wie sie enttäuscht werden. Konstanz.

Downie, Jr., Leonard, Michael Schudson (2009): The Reconstruction of American Journalism. Columbia University Graduate School of Journalism. www.cjr.org/reconstruction/the_recon struction_of_american.php [30.12.2010].

Fengler, Susanne, Stephan Ruß-Mohl (2005): Der Journalist als „Homo oeconomicus“. Konstanz.

Göbel, Elisabeth (2002): Neue Institutionenökonomik. Konzeption und betriebswirtschaftliche Anwendungen. Stuttgart.

Freidson, Eliot (1983): The Theory of Professions: State of the Art. In: Dingwall, Robert, Philip Lewis (Eds.), The Sociology of the Professions: Lawyers, Doctors and Others. London, 19-37.

Freidson, Eliot (2001): Professionalism. The Third Logic. Cambridge, U.K.

Haller, Michael (2004): Die zwei Kulturen. Journalismustheorie und journalistische Praxis. In: Löffelholz, Martin (Hg.), Theorien des Journalismus. Wiesbaden, 129-190.

Heinrich, Jürgen (2001): Medienökonomie. Band 1: Mediensystem, Zeitung, Zeitschrift, Anzeigenblatt. 2. Auflage. Wiesbaden.

Hess, Charlotte, Elinor Ostrom (2007): Introduction: An Overview of the Knowledge Commons. In: Dies. (Eds.), Understandig Knowledge as a Commons. From Theory to Practice. Cambridge, Mass., London, 3-26.

Jäger, Wieland, Uwe Schimank (Hg.) (2005): Organisationsgesellschaft. Facetten und Perspektiven. Wiesbaden.

Kepplinger, Hans-Mathias (2004): Problemdimensionen des Journalismus. Wechselwirkung von Theorie und Empirie. In: Löffelholz, Martin (Hg.), Theorien des Journalismus. Wiesbaden, 87-105.

Kiefer, Marie Luise (2010): Journalismus und Medien als Institutionen. Konstanz.

Kiefer, Marie Luise (2005): Medienökonomik. 2. Auflage. München, Wien.

Klatetzki, Thomas (2005): Professionelle Arbeit und kollegiale Organisation. Eine symbolisch interpretative Perspektive. In: Klatetzki, Thomas, Veronika Tacke (Hg.), Organisation und Profession. Wiesbaden, 253-283.

Klatetzki, Thomas, Veronika Tacke (Hg.) (2005): Organisation und Profession. Wiesbaden.

Kohring, Matthias (2004): Vertrauen in Journalismus. Theorie und Empirie. Konstanz. 
Kranich, Nancy (2007): Countering Enclosure: Reclaiming the Knowledge Commons. In: Hess, Charlotte, Elinor Ostrom (Eds.), Understanding Knowledge as a Commons. From Theory to Practice. Cambridge, Mass., London, 85-122.

Kühl, Stephan (2006): Die Professionalisierung der Professionalisierer? Das Scharlatanerieproblem im Coaching und der Supervision und die Konflikte um die Professionsbildung. Working-Paper 4. www.uni-bielefeld.de/soz/organisationssoziologie/pdf/p0406.pdf [30.12.2010].

Levine, Peter (2007): Collective Action, Civic Engagement and the Knowledge Commons. In: Hess, Charlotte, Elinor Ostrom (Eds.), Understanding Knowledge as a Commons. Cambridge, Mass., London, 247-275.

Littek, Wolfgang, Ulrich Heisig, Christel Lane (2005): Die Organisation professioneller Arbeit in Deutschland. Ein Vergleich mit England. In: Klatetzki, Thomas, Veronika Tacke (Hg.), Organisation und Profession. Wiesbaden, 73-118.

Luhmann, Niklas (1996): Die Realität der Massenmedien. 2. Auflage. Opladen.

Meyer, John W., Ronald L. Jepperson (2005): Die Akteure der modernen Gesellschaft: Die kulturelle Konstruktion sozialer Agentschaft. In: Meyer, John W., Weltkultur. Wie die westlichen Prinzipien die Welt durchdringen. Herausgegeben von Georg Krücken. Frankfurt, 47-84.

Mieg, Harald (2003): Problematik und Probleme der Professionssoziologie. In: Mieg, Harald, Michaela Pfadenhauer (Hg.), Professionelle Leistung - Professional Performance. Konstanz, 11-46.

Mögerle, Ursina (2009): Substitution oder Komplementarität? Die Nutzung von Online- und PrintZeitungen im Wandel. Konstanz.

Neidhardt, Friedhelm (1994): Öffentlichkeit, öffentliche Meinung, soziale Bewegungen. In: Ders. $(\mathrm{Hg})$, Öffentlichkeit, öffentliche Meinung, soziale Bewegungen. Sonderheft 34 der Kölner Zeitschrift für Soziologie und Sozialpsychologie, 8-41.

Neverla, Irene (1998): Die verspätete Profession: Journalismus zwischen Berufskultur und Digitalisierung. In: Duchkowitsch, Wolfgang u. a. (Eds.), Journalismus als Kultur. Wiesbaden, 53-62.

Olson, Mancur (1992): Die Logik des kollektiven Handelns. Kollektivgüter und die Theorie der Gruppen. 3. Auflage. Tübingen.

Ortmann, Günther (2005): Organisation, Profession, Bootstrapping. In: Klatetzki, Thomas, Veronika Tacke (Hg.), Organisation und Profession. Wiesbaden, 285-298.

Ostrom, Elinor (1999): Die Verfassung der Allmende. Jenseits von Staat und Markt. Tübingen.

Ostrom, Elinor (2005): Understanding Institutional Diversity. Princeton, Oxford.

Ostrom, Elinor, Charlotte Hess (2007): A Framework for Analyzing the Knowledge Commons. In: Hess, Charlotte, Elinor Ostrom (Eds.), Understandig Knowledge as a Commons. From theory to Practice. Cambridge, Mass., London, 41-81.

Peters, Bernhard (2007): Der Sinn von Öffentlichkeit. Hrsg. von Hartmut Weßler. Frankfurt/M.

Piper, Nikolaus (2010): Meinen statt wissen. Was der jüngste Bericht zur Lage der amerikanischen Medien über die Zukunft des Journalismus aussagt. In: Süddeutsche Zeitung vom 27./28. März, 23.

Pöttker, Horst (2003): Zahnloser Tiger? Plädoyer für wirksame Selbstkontrolle des Journalismus im Dienste der Kommunikationsfreiheit. In: Langenbucher, Wolfgang R. (Hg.), Die Kommunikationsfreiheit der Gesellschaft. Die demokratischen Funktionen eines Grundrechts. Sonderheft 4 der Publizistik, 379-384.

Priddat, Birger P. (1996): Die Zeit der Institutionen. Regelverhalten und rational choice. In: Priddat, Birger P., Gerhard Wegner (Hg.), Zwischen Evolution und Institution. Marburg, 11-34.

Richter, Peter (2009): Ökonomisierung als gesellschaftliche Entdifferenzierung. Eine Soziologie zum Wandel des öffentlichen Sektors. Konstanz.

Röper, Horst (2010): Zeitungen 2010. Daten zur Konzentration der Tagespresse. In: Media Perspektiven Nr. 5, 218-234.

Rühl, Manfred (1980): Journalismus und Gesellschaft. Bestandsaufnahme und Theorieentwurf. Mainz.

Rühl, Manfred (2004): Theorie des Journalismus. In: Burkart, Roland, Walter Hömberg (Hg.), Kommunikationstheorien. Ein Textbuch zur Einführung. 3. Auflage. Wien, 117-140. 
Rühl, Manfred (2008): Beobachtete Paradoxien. Über den Wandel weltgesellschaftlicher Journalistik. In: Pörksen, Bernhard, Wiebke Loosen, Armin Scholl (Hg.), Paradoxien des Journalismus. Theorie - Empirie - Praxis. Wiesbaden, 567-579.

Saxer, Ulrich (2007): Politik als Unterhaltung. Zum Wandel politischer Öffentlichkeit in der Mediengesellschaft. Konstanz.

Schröder, Guido (2008): Positive Medienökonomik. Institutionenökonomischer Ansatz für eine rationale Medienpolitik. Baden-Baden.

Searle, John R. (2005): What is an Institution? In: Journal of Institutional Economics 1, 1, 1-22.

Siebenhaar, Hans-Peter (2010): Pay oder Pleite? Die neue Ökonomie der Medien. In: Tendenz. Magazin der Bayerischen Landeszentrale für neue Medien, 6-11.

Stichweh, Rudolf (2005): Wissen und die Professionen in einer Organisationsgesellschaft. In: Klatetzki, Thomas, Veronika Tacke (Hg.), Organisation und Profession. Wiesbaden, 31-44.

Suber, Peter (2007): Creating an Intellectual Commons through Open Access. In: Hess, Charlotte, Elinor Ostrom (Eds.), Understanding Knowledge as a Commons. From Theory to Practice. Cambridge, Mass., London, 171-208.

Tacke, Veronika (2005): Schulreform als aktive Deprofessionalisierung? Zur Semantik der Lernenden Organisation im Kontext der Erziehung. In: Klatetzki, Thomas, Veronika Tacke (Hg.), Organisation und Profession. Wiesbaden, 165-198.

Türk, Klaus, Thomas Lemke, Michael Bruch (2006): Organisation in der modernen Gesellschaft. Eine historische Einführung. 2. Auflage. Wiesbaden.

Weichert, Stephan, Leif Kramp (2009): Das Verschwinden der Zeitung? Internationale Trends und medienpolitische Problemfelder. Studie im Auftrag der Friedrich-Ebert-Stiftung. Berlin.

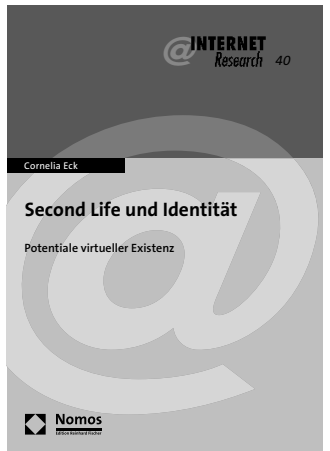

Bitte bestellen Sie im Buchhandel oder versandkostenfrei unter $\downarrow$ www.nomos-shop.de

\section{Second Life und Identität}

Potentiale virtueller Existenz

Von Cornelia Eck

2011, 333 S., brosch., 39,-€

ISBN 978-3-8329-6324-8

(Internet Research, Bd. 40)

Eignet sich die virtuelle Welt Second Life als postmoderne Identitätsbühne? In ihrem Buch „Second Life und Identität" nähert sich die Autorin Cornelia Eck dieser Frage aus verschiedenen identitätstheoretischen und empirischen Perspektiven. Im Zentrum steht dabei die Auswertung einer umfangreichen wissenschaftlichen Befragung von 565 deutschsprachigen Second-Life-Nutzerinnen und -Nutzern.

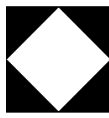

